\title{
Public Health Responding to the Epidemic of Alzheimers Disease
}

\author{
David P Hoffman DPS, CCE* \\ Ethics and Health Policy, Maria College, 700 New Scotland Ave. Albany, NY 12208, USA
}

${ }^{*}$ Correspondence author: David P Hoffman DPS CCE, Associate Professor, Ethics and Health Policy, Maria College, 700 New Scotland Ave. Albany, NY 12208 Tel:+5188612582; Email: dhoffman@mariacollege.edu

Received: August 22, 2019; Accepted: August 28, 2019; Published: September 04, 2019;

In the course of my career I've written frequently about the needs of caregivers of people with dementia and the urgency for public health to address their health and stress issues to avoid a human and health crisis in our culture. Recent publications by the Centers for Disease Control and the Alzheimers Association highlight that these issues are even more critical [1,2]. The stress of caring for a vulnerable person with dementia, or other person with special needs is a major risk factor for developing numerous physical and emotional chronic conditions.

Let's be clear, we're experiencing an aging population trend and commensurate increase in age related conditions. Chief among these are the various forms of dementia that constitute the epidemic I reference above. Alzheimers disease is the most common form of dementia, and we now recognize it often appears along with other forms of dementia and related conditions. During the course of these diseases one aspect we humans lose is independence, this leads to the necessity of persons to care for those with dementia for their health, well-being and everyday needs. Much has been written about the empathy of these people; sometimes referring to them as "angels", what I want to focus on today is the very real risk to their health and mental health that comes with the role of caregiver. The nature of this role often leads to risky health behaviors such as sedentary lifestyle, poor nutrition, social isolation, and overuse and abuse of substances such as alcohol or prescription drugs. Additionally, economic strain frequently occurs with the all too common absenteeism or presenteeism at/from work, additional costs related to obtaining healthcare for the patient with dementia, and selfless acts of putting the patient before the caregiver's own needs. These stresses can also complicate otherwise strained relationships and result in increasing risks to health and mental health.

This important public health issue cuts both ways. First the person with needs who requires care is at risk, and second the role of caregiver causes risk as well. Given that this phenomenon relates to millions of Americans alone, the impact around the globe is multiples of the recently published data. In the US health and well-being of informal caregivers is in many ways the backbone of the system of long-term care. This includes the healthcare system and what serves as the system of long-term care services and supports. The everyday needs of those requiring care become dependent on a population at high risk of chronic conditions both physical and emotional [3].
Recent CDC data highlight that over $40 \%$ of caregivers report having two or more chronic diseases; $14.5 \%$ of caregivers report experiencing 14 or more mentally unhealthy days in the last month; and $36.7 \%$ report getting insufficient sleep. Given that these are selfreported statistics, arguably the actual numbers may be higher [1]. As I have frequently stated in the past [4-7], those of us in caring professions have a moral and ethical responsibility to find ways to support these hardworking caregivers and policy-makers must act on their responsibility to create policies and systems that enable caregivers to perform their duties without compromising their own health and economic well-being.

We know that caregivers with knowledge, access to support systems, ability to engage socially, and resources to make healthy choices live healthier and more fulfilling lives. They are also more likely to have the energy to continue caring for their vulnerable loved one, manage other family responsibilities (i.e., sandwich generation members - those caring for children and an aging parent), and maintain their occupation, thus limiting collateral impacts across families and communities.

One key element to ensuring that a caregiver has the information and access to support is an actively engaged health care provider - either the caregiver's health care provider or the provider for the person receiving care. It should be both, but either should take some responsibility as a preventive measure. Today some practical suggestions for consideration include:

1. In clinical and human service encounters we have a professional responsibility to tune in to the well-being of people who are caregivers. This tuning in can and should happen both informally ("So how are you doing today?") and formally ("Let's check your vital signs while you are here."). These vital signs should include assessing the caregiver's sleep, nutrition, physical activity, work/life balance, use of alcohol, and access to support services, including respite.

2. Assessing knowledge and coping skills to reduce caregiving burden on multiple fronts. In the case of progressive disease like dementia, for instance, we can assess whether there is a plan for how to manage the increasing physical demands of that role.

3. Plain language resources on respite and other resources should be repetitively available (in healthcare offices, social service settings, religious communities). 
4. Tips for caregivers should be similarly available (how to communicate, coping with common challenges such as hearing loss and limited mobility. Tips on managing common costs and available discounts and supports (for phone, cable, trash removal, food delivery).

5. Expand proven strategies such as the New York State program referenced below and Naturally Occurring Retirement Communities that can provide formal and informal supports and the key service of having multiple sources of "checking in" to reduce the feeling of isolation [4].

These are just a few suggestions, the critical issue today is the need for action on this challenge, especially where other additional challenges are present. In previous articles there have been many calls for action on these questions [6], including a call for a national plan. Today my call is for a systemic approach to address the health of vulnerable members of society who require care and the integral informal caregivers who attempt to address these needs in every community across the country. In my former role, with support of advocates like the Alzheimers Association and key policymakers, New York State invested significantly in both Centers of Excellence to educate professionals and provide high quality clinical services across the state and various Caregiver Support Programs strategically located to be accessible statewide. This program was built because using data like that from CDC referenced below, we were able to show that early diagnosis, aggressive quality clinical care and an array of proven caregiver support activities in addition to being lifesaving to patients and caregivers could also be cost effective. I recognize this doesn't solve all the issues raised here, but this huge step forward can provide lessons for nations, states and other jurisdictions. Evaluation data on the program prepared by NYSDOH with a team at the Albany School of Public Health led by Dr. Mary Gallant can be reached at: https:// www.health.ny.gov/health_care/medicaid/redesign/mrt_8004.htm

A new set of materials and data I reference above from the Centers for Disease Control and Prevention related to Alzheimer's disease, including background information as well as additional suggested action steps for a variety of audiences can be found here:

- What is Alzheimer's disease?

- Road Map for Indian County

- Road Map for State and Local Public Health

- Caregiving - A Public Health Priority

- Caregiving for Family and Friends-Data for Public Health Action

- Data Portal-Alzheimer's Disease and Healthy Aging

\section{References}

1. https://www.cdc.gov/aging/caregiving/caregiver-brief.html

2. Alzheimer's Association (2019) Alzheimer's Disease Facts and Figures. Alzheimers Dement 2019 15: 321-387.

3. Hoffman D, Zucker H (2016) A Call to Preventive Action by Health Care Providers and Policy Makers to Support Caregivers. Prev Chronic Dis 13: 160233. [crossref]

4. Masotti PJ, Fick R, Johnson-Masotti A, MacLeod S (2006) Healthy Naturally Occurring Retirement Communities: A Low-Cost Approach to Facilitating Healthy Aging. Am J Public Health 96: 1164-1170. [crossref]

5. Hoffman D (2014) Alzheimers Disease Legislation and Policy, Now and in the Future. Health Aff (Millwood) 3: 561-565. [crossref]
6. Khachaturian AS, Hoffman DP, Frank L, Petersen R, Carson BR, et al. (2017) Zeroing out preventable disability: Daring to dream the impossible dream for dementia care: Recommendations for a national plan to advance dementia care and maximize functioning. Alzheimer's Dement 13: 1077-1080. [crossref]

7. Hoffman D (2015) Looking at The Future of Alzheimers Disease Policy. Health Affairs Health Policy Lab JULY 14, 2015.10.1377/HBLOG20150714.049333
Citation:

Hoffman D (2019) Public Health Responding to the Epidemic of Alzheimers Disease. Ageing Sci Ment Health Stud Volume 3(3): 1-2. 